\title{
Architectures for Rapid Prototyping of Model-based Robot Controllers
}

\author{
Basilio Bona, Marina Indri, and Nicola Smaldone \\ Dipartimento di Automatica e Informatica \\ Politecnico di Torino \\ Corso Duca degli Abruzzi 24, 10129 Torino, Italy \\ basilio.bona@polito.it,marina.indri@polito.it,nicola.smaldone@polito.it \\ http://www.ladispe.polito.it/robotica/Labrob/Index.htm
}

\begin{abstract}
Rapid prototyping (RP) in control design can be defined as a computer-assisted process aimed at recursively validate dynamic models of complex plants and mechatronic systems and/or design and test digital control algorithms for real-time applications.

Rapid prototyping of digital control algorithms requires integrated hardware/software architectures, allowing fast and systematic interactions between the algorithmic design phase and the experimental testing. The design phase is performed with the support of a computer aided control design environment, where simulations are performed on accurate models of the specific equipment under investigation; after that, a rapid transfer of the algorithm on the target hardware is necessary to validate it experimentally. It is therefore necessary to have a complete prototyping environment, where different controller blocks are readily available, with structure and parameters easily modifiable to be tested on the simulated plant and downloaded on the target hardware platform for real-time validation.

The present Chapter introduces the state-of-the art on RP, critically surveys and discusses general issues related to both HW and SW aspects that are at the basis of RP; furthermore it describes in some details the solution implemented by the authors at the Experimental Robotics Laboratory of Politecnico di Torino. A test-case, devoted to the problem of modelling and compensation of nonlinear friction in rotating robot arms is presented.

Finally, a critical appraisal of the proposed solution, in the light of the gained experience, is discussed and future developments are pointed out.
\end{abstract}

\section{Introduction and State of the Art}

Prototyping can be defined as: "A type of development in which emphasis is placed on developing prototypes early in the development process to permit early feedback and analysis in support of the development process" [1]. The implementation of a prototype starts from an idea which is then developed in a project phase, where several alternative solutions are considered to achieve the desired functionalities and specifications. Design relies on technical competence and objectives; several tools can help the designer to practice that competence and to define the objectives in details. Using a personal computer (PC) in the prototyping phase as a replacement of traditional technical tools is a common practice today. One of the most important features of the PC is the possibility of virtualizing the objects and the procedures to build them. For example, in architectural design, the computer graphics allows to 
visualize the whole inhabited environment in some details and to verify the design hypothesis formulated by the architect; mechanical engineers can try to combine some graphical objects representing mechanical parts, starting from the drawings of such parts, to test their functionalities.

A major interests in prototyping derives from the possibility of knowing the influence of design solutions before the final production phase. In manufacturing, where small technological objects are often produced in large quantities, prototyping allows to build the trial version in order to verify a subset of functionalities, before the cost of possible design errors grows up due to the large number of manufactured parts. In such a situation the PC can be useful as it automates the large number of procedures involved in the construction of the prototype.

In more general terms, the prototyping process makes easier the application of specific methodologies from different technological fields embedded into a real or virtual instance of a product.

In the last few years these aspects are becoming one of the major issues in control design for advanced mechatronic equipments and robotics $[7,9,10]$.

In the field of industrial robotics there are several kinds of prototyping processes; a manipulator embodies different technologies and competences: mechanical, electronic and electrical issues merge with automatic control and computer science competencies for a satisfactory design of whole machine.

In the present work we discuss prototyping issues and architectures for control and supervision of industrial robots; the aim of prototyping is often the implementation of new control algorithms or architecture allowing better performances at lower costs in well defined operating conditions.

An objective only partially reached today is the so called rapid prototyping, i.e. a methodology which allows to go in a short time and with limited costs from the general idea to the realizable solution. After the prototype design is tested on the real equipment, one must be able to repeat cyclically the same procedure with only a marginal additional effort.

The prototyping process consists of a set of phases, often technologically very different; this fact complicates in a remarkable way the transmission of information, especially when formalisms and techniques used before the PC advent are involved. So, rapid prototyping must be based on a friendly and homogenous development environment, which should allow the designer to concentrate on conceptual problems freeing him/her from the tedious practical aspects involved in the progression from the idea to the prototype.

The PC plays a major role hosting the interactive environment allowing to develop many of the rapid prototyping process phases, as, for example:

- to model the controlled electromechanical parts;

- to design the control laws and the machine supervision software;

- to simulate the effects of the control algorithms;

- to automate the transition from the design formalism to the implementation and adaptation to the machine execution;

- to manage the interaction between designer and test machine. 
The last point introduces a problem that is common to all the environments where automatic controlled evolution of physical phenomena is needed, i.e. realtime requirements.

Industrial robots are supplied with a controller cabinet containing hardware and software systems for control and supervision. Due to industrial secrecy, safety requirements or, sometimes, technological backwardness, these systems are closed to modification by the customers. On the other hand a controller presents many critical aspects due to the simultaneous presence of components with contrasting real-time requirements.

The user of a prototyping system should have at least the possibility to interface the original control environment, and in many cases partially or totally substitute it; therefore it is necessary to pay attention to the real-time issues in order to avoid interferences with the native architecture, especially when it is necessary to replace important functionalities.

In the following Section some concepts related to the real-time interaction between PC and controlled mechatronic equipments will be introduced; basic definition will be briefly presented, and methodologies will be described. Particular attention will be paid to the real-time requirements of rapid prototyping systems.

\section{Rapid Prototyping}

For each specific "product" the prototyping process requires a test platform, where it is possible to investigate the characteristics and the potentiality of several alternative solutions, before arriving to the final product release. In the field of control systems for industrial robots, the product usually consists in control algorithms for robotic axes or software for machine supervision and man-machine interface; at the same time this platform allows to deal in an efficient manner with the plant modelling too, using simple simulation and test procedures.

The electronics of a control system ready to be commercialized is the result of optimization in workspace, performances, reliability and costs. It is advisable to test the functionalities of the design ideas using standard and re-usable components; on a single prototype costs are often greater than those of the final product, but the possibility of searching a solution without worrying about non-functional constraints (power consumptions, space, reliability, etc.) and the simplification of more complex problems should be considered. Furthermore, the product will be often sold in large quantities, making the prototype costs negligible. In other cases the costs may be disregarded because the designer is interested only in a limited number of performances with respect to those of the actual product.

The test platform usually consists of a software environment representing the plant and the control components according to some conceptual metaphor, often a graphical one. One of the main characteristics in this environment is the possibility to simulate the functionalities of the system both on time basis and on logical basis.

Often the prototyping software is coupled with the real plant using configurable electronic boards. The advantages of this methodology result from two fundamental 
factors: a) the extreme configurability of the software environment, which allows to modify all the design parameters and to foresee the possible consequences; $b$ ) the adaptability of the prototyping electronics composed by standard and modular components.

The possibility of simulating the control algorithms avoids potentially dangerous situations for the equipments, which, on the other end, may be inaccessible for the tests. Indeed, the complexity of some plants requires a separation of the design into subsystems, where each one needs to be tested independently from the others. This procedure may be impossible for industrial manipulators with many degree of freedom due to the highly coupled nature of the kinematic chain.

In industrial manipulator prototyping the possibility of simply formalizing the mathematical models for kinematics and dynamics, which will be used in the simulation software, is one of the more interesting features; in this manner all the control algorithms can be tested and interfaced with these models. Models must be enough refined to describe all the phenomena judged critical for control; for example, the model can take into account joint frictions, disturbances and parameters uncertainty, but not address the elasticity issues, if these are not critical.

When the robot is accessible and it is possible to interface it with prototyping system, the test phase can be managed directly from the development and simulation environment. The prototyping system is sometimes called Host and it is independent from the constraints due to the plant interfacing, thanks to the presence of another computer, the Target, which supervises to the interaction with the manipulator. The Host interacts in asynchronous mode with the Target to set the test execution modalities and to monitor its progression; the Target receives commands and data from the Host and, through the interface electronics, controls the robot in real-time. If the plant is particularly articulated, then a multi-Target system, possibly with each Target synchronized with the others, can be necessary, whereas the Host can remain unique. In some particular cases, a single system can be used as both development and plant interaction environment.

The Host environment is often called a CAD system since it allows a "Computer Aided Design"; in particular, for automatic controls it is named CACSD, from "Computer Aided Control System Design". The Target environment can have one of the possible architectures suitable for real-time requirements; these architectures and some basic concepts are presented in the following Section, on the basis of quantity and complexity of tasks concentrated on it.

\subsection{Real-Time systems}

The key issue of real-time systems for automatic control is the proper interaction with physical phenomena representing real processes.

Interaction, performed by a computer program, takes place through signals, whose time history is characterised by a dominating time constant. Because of the limits on the available resources, it is necessary to select the relevant time constants, in order to update the knowledge of input signals, and to recompute the output commands to control the process as requested. 
A real-time system uses software structures called Tasks to perform this kind of interaction while complying with the assumed time constraints.

These Tasks are often characterized by different time constants and have to be executed within the same time window by the same computer; for this reason the software needs to share the calculus resources between all these activities.

A Task with hard real-time requirements must complete its job strictly inside the time interval planned on the basis of the control criticalities, in order to avoid the total failure of the process. So it is necessary to be certain, using some procedures or exhaustive simulations, that the system will not infringe the time constraints of that Task [2].

In a Task with soft real-time requirements, instead, lacks the claim of never infringe the time constraints, or warrants it in some statistical sense, e.g. in the "majority of cases": infringing the time constraints for the soft components is accepted as an unsubstantial degradation of the system functionalities.

Both these kinds of Tasks can coexist in a robot control system; for example, the closed loops of the control axis or the emergency procedures related to the limit switches activation are hard real-time Tasks, whereas signaling non-critical anomalies or refreshing the control workstation graphical user interface are considered soft real-time Tasks.

In this context an implicit hypothesis is assumed: all Tasks are arranged on a unique computer and the capacity of parallel execution of different connected Tasks is called multitasking. The hard real-time requirements fulfillment can be guaranteed avoiding to use hardware and software components which can bring to non-deterministic behaviors; or, according to more refined techniques, dynamically checking that each new Task will have the possibility, on the basis of the current workload, of completing its jobs and respecting its time constraints.

\subsection{Architectures, characteristics and requirements of robot prototyping systems}

The hardware architecture of a simple Commercial-Off-The-Shelf (COTS) computer is shown in Figure 1.

RAM and CPU are the fundamental resources, and various Tasks use them according to defined specifications and procedures, aimed to implement a correct multitasking for real-time requirements. To allow the Task interaction with the outer world and the physical phenomena under control, additional components, called Input/Output devices, are needed. These components are essentially electronic devices acting as bit converters to and from electrical external signals. A typical example of I/O devices for automatic control systems are DACs, Digital-to-Analog Converters, and ADCs, Analog-to-Digital Converters.

Communication between CPU, RAM and I/O devices is carried out through a system Bus, which becomes a shared resource and, as such, requires a sharing mechanism. It should be noted that each time resources are shared, the time constraints and the deterministic behavior dictated on the real-time system are threatened: if two 
Fig. 1. A very simple elaboration system

real-time Tasks need the same data bus, the first which obtain it can delay or prevent the other Task to complete its job in time.

When a Task sends a command to an I/O device, that device will spend some time doing it. To optimize the CPU use for as many Tasks as possible, a Task freezing technique is adopted when it starts an I/O procedure and is waiting data from the device; this characteristics is called preemption. Preemption allows other Tasks in ready status to use the CPU; when the device ends its job and data are ready to be transferred, it notifies its state using a new signal called Interrupt [2-5].

The Interrupt is usually managed by an integrated circuit, which sends the relevant information to the CPU. The Interrupt signals from each device are received by some devoted pins of the CPU, the Interrupt request (or IRQ) pins; the CPU decides which device to serve first on the basis of a priority mechanism and the right Interrupt Service Routine (ISR) takes care of the event. Then the Task which started the I/O procedure is waked up as soon as possible to complete its job and to free the resources it is using.

This procedure is sketched in Figure 2. $T_{L}$ is the Interrupt latency time; it is an important parameter, since it allows to evaluate the responsiveness of a real-time system to an event.

Fig. 2. Interrupt mechanism 
Real-time software architectures can be divided according to the complexity and the speed of response guaranteed to the Tasks. In the following Sections a brief survey of the two principal software architectures is presented; they both allow to manage the interaction with $\mathrm{I} / \mathrm{O}$ ports and with the user, showing the right compromise between simplicity and adequacy of the components and real-time characteristics.

Round-robin with Interrupt. The ISR of each I/O device takes part in the signal and protocol handling and reserves the data exchange service; an infinite loop Task looks after the requesting devices [3].

Note that the failure in one of the devices could lock the Task for a long time. Furthermore, the global variables accessible by the ISRs and by the Task must be protected; in fact, the ISRs could modify these variables in any instant and could give back to the Task a different environment without the Task knowing it.

Real-Time Operating System. Maximum flexibility, but also maximum complexity, can be obtained using a real-time operating system (RTOS). ISRs and Tasks can be executed in parallel and according to fixed priorities; a software component called scheduler intervenes when particular "events" occur in the system, to switch the CPU control from a Task to a new one. This is the so called processes model [5], in which an ISR or a Task represents a process, with all its data, its executable code and the system identification parameters. Each process carries out its own job sequentially and, virtually, disposes of a dedicated CPU; whereas actually, the only CPU in the system (in the mono-processor systems) is shared on time basis between all processes in execution.

\subsection{Prototyping systems for robotics}

Robotic systems are composed by several mechanical parts moved by means of electrical drives. In particular, a robot has several arms and joints arranged in a complex kinematic chain; the entire structure is moved combining the motion of each joint. A supervision/control system takes care of the activation "rules" for each drive so that the articulated structure carries out the desired tasks [14].

Command signals from the computing system to the actuators are the result of appropriate computation on data coming from sensors, the so called feedback control. This modality is critical from a computational point of view because it must be characterized by a reasonable knowledge of execution times. In such cases the real-time requirements play an important role.

In this Chapter our interest will be concentrated on these real-time systems, which represent a particularly complex branch of mechatronics, and on their prototyping.

The supervision of these systems requires the execution of several activities with hard or soft real-time issues; a list of these activities can be the following:

- position control of single joints;

- trajectory planning for coordinate movements; 
- enabling phases and emergency control;

- man-machine interface.

At a higher task level, with respect to axis control, it is necessary to decide how a desired tip movement in the working space can be obtained and to send correct guidelines to each motor. Movements must take into consideration all the enabling requirements of the individual components and the safety for the operators and the machine. Anomalous behaviors, like unexpected collisions, motor current overloads or joint limit switch activations, need to be detected as soon as possible.

The system could also provide some sort of man-machine interaction: the operator may need a simple graphical interface to configure the functionalities of the manipulator tasks or use some more complex integrated diagnostic tool.

Therefore there are many complex and concomitant Tasks to execute; a single computer can manage all of them or it could be appropriate to devote a computer to the low level machine handling, leaving the soft-real-time Tasks to a separate one.

In the following Section such architectures are briefly described.

Hardware architectures. When prototyping is concerned, i.e. when systems usually do not work in "extreme" conditions and with heavy workloads, the set of available hardware components can include general purpose devices. In the last years the trend has been to use commercial-off-the-shelf (COTS) components due to their low costs and tested reliability.

The Host PC must be able to run the development tools, and to perform simulation processes of various kind, which is the most important assignment of a prototyping system.

The Target machine is often less powerful, but equipped with I/O boards to interact with the plant. Conversion speed of the on-board electronics, the communication bus between CPU and boards and Interrupt latency are typical bottlenecks for the Target.

Software architectures. In the context of control for robotic systems, Tasks can be classified as synchronous (or periodic) and asynchronous (or aperiodic). A reliable internal mechanism to provide a timing base for synchronous events is needed. The asynchronous events occuring during the normal activities are dealt with in the spare time between the synchronous events.

Clock-driven architectures [2] are ideal candidates for this type of Tasks: the presence of periodic Tasks $T_{i}$, having well defined real-time execution characteristics is contemplated. An interrupt related to clock signals wakes up the scheduler according to the period of each Task. A Task $T_{i}$ is defined by two parameters: the period $P_{i}$ and the execution time $e_{i}$; it is assumed that $T_{i}$ finishes its job before the end of its period, to guarantee the execution in the next period. The $P_{i}$ can be different if the arrangement of $T_{i}$ is done according to an hyperperiod equal to the least common multiple between all $P_{i}$. The Tasks scheduling must be arranged to allow 
the execution of all Tasks, according to their periods, into a unique hyper-period which will be repeated indefinitely.

This type of scheduling gives origin to inactive intervals, during which the aperiodic Tasks can be executed; these Tasks have soft-real-time characteristics and can treat "normal" situations.

There are also sporadic Tasks, which are usually devoted to react to unexpected events with hard real-time characteristics.

The clock-driven architecture can be implemented using both round-robin with interrupt and RTOS.

\subsection{Prototyping tools}

The CAD environment allows to represent the manipulator kinematics and dynamics by some sort of formalism. Simulink ${ }^{\circledR}$ and Matlab ${ }^{\circledR}$ from The Mathworks, Inc. ${ }^{\circledR}$ are CAD tools widely used in research and design of control systems. Simulink ${ }^{\circledR}$ allows to assemble system parts according to a graphical block formalism. In Simulink ${ }^{\circledR}$ it is possible to include event-driven process logic using the Stateflow ${ }^{\circledR}$ tool; this instrument is based on finite state machine theory. A Stateflow ${ }^{\circledR}$ diagram is composed by blocks representing states, and the simulator passes from one to another when some specified event happens. These events are associated to oriented edges linking the state blocks and labels specifying conditions and, possibly, actions. Both Mealy and Moore paradigms (actions associated to transitions and action associated to states, respectively) are supported. A Stateflow ${ }^{\circledR}$ diagram included in a Simulink ${ }^{\circledR}$ diagram can implement conditions and constraints on the execution of the overall simulation.

Automatic code generation. Crucial to prototyping is the implementation of control and supervision algorithms on the actual controller: it is necessary to translate the block formalism into a high level language, usually $\mathrm{C}$ or $\mathrm{C}++$. In order to obtain a Target processor executable it is necessary to perform program compilation by a cross-compiler residing on the Host PC. The program is then transferred and run on the Target PC using software tools resident on the Host, able to manage, monitor and in case also debug the testing progression.

The real-time software architectures described above are the starting point for building the control structure; to reduce the error possibilities and to cut the prototyping process time, automatic code generation can be used. This process is called rapid prototyping: Real-Time Workshop ${ }^{\circledR}$ (RTW) and Stateflow coder ${ }^{\circledR}$ are the tools which translate each block and the finite state machines in a programming language specified by the user, usually in $\mathrm{C}$. There are also some rules to define how to code block relations and organization.

This last characteristic is interesting for real-time programmers because it gives the possibility to choose the resulting software architecture. Two architectural models, already described, are available:

- round-robin with Interrupt model; 
- processes model based on RTOS.

Both architectures can manage multitasking; in the following Sections two implementation example are described using pseudocode.

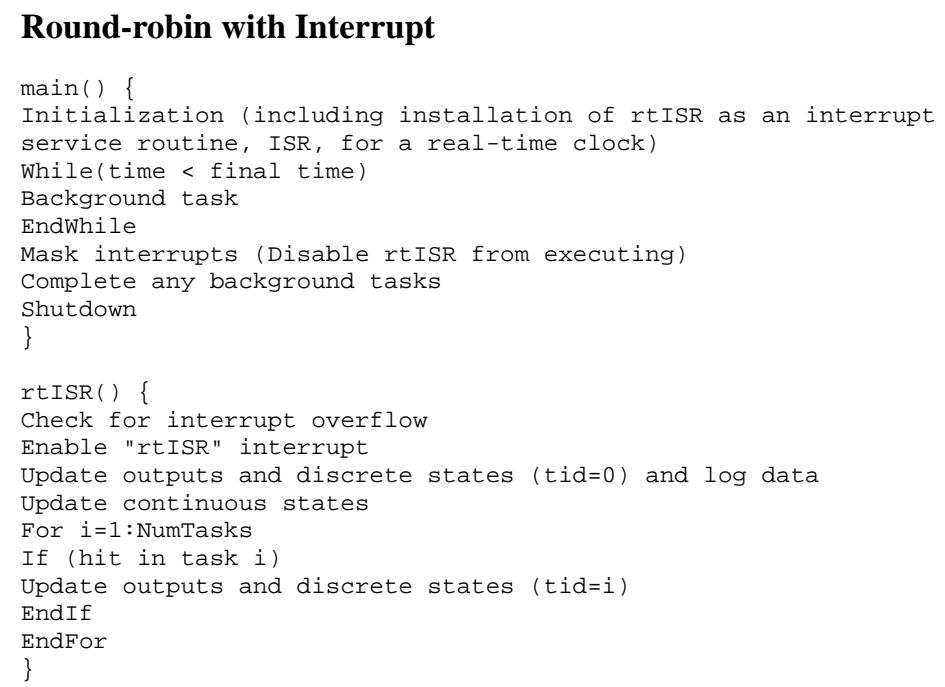

The rtISR procedure is executed when an Interrupt is generated by a clock, with a cadence equal to the fastest sampling time present in the model. Its structure is similar to the simulation mechanism: output update, discrete states update, continuous states integration (if present, the ISR execution period equals the integration step). Multitasking is built imposing multiple sampling times with respect to the basic Task $(\mathrm{t} i \mathrm{~d}=0)$, so that for each Interrupt cycle the states having the sampling time tick in that instant are updated. During rtISR inactivity period a Background Task with non-real-time jobs is executed. The whole mechanism is started by the main routine which organizes the real-time clock, the ISR and the Background execution cycle; the same routine ends the execution, masking the Interrupt signal and completing the Background.

\section{Multiprocess with RTOS primitives}

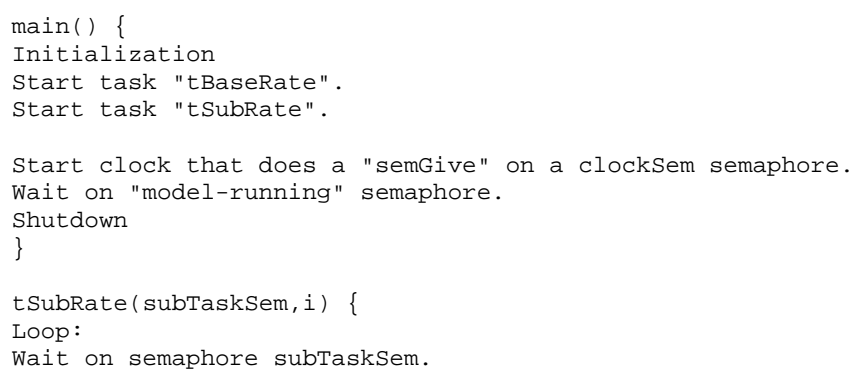




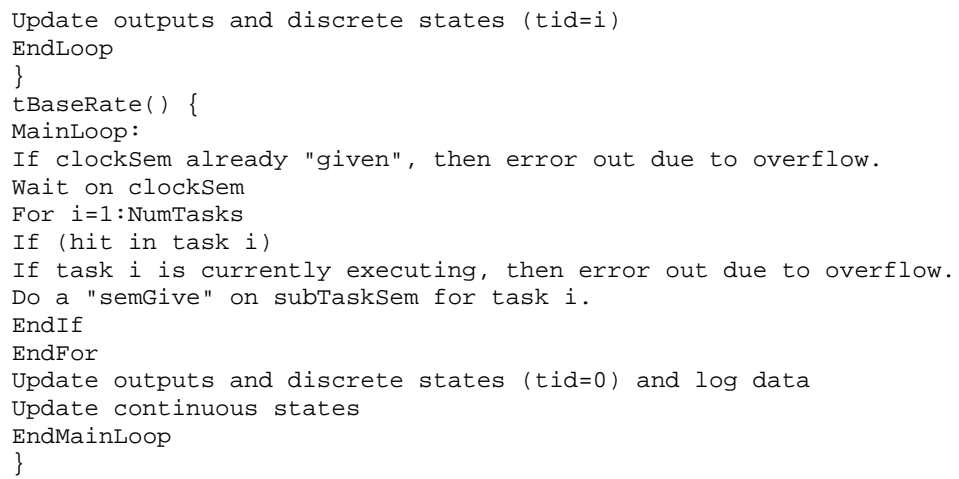

In this case the model is executed using some typical RTOS primitive: processes creation and start with fixed priority, and Task synchronization by means of semaphores. The main procedure creates a tBaseRate process having the highest priority, i.e. waked up by the fastest clock period of the model. More tSubRate processes with multiple sampling time with respect to tBaseRate are created, each one with decreasing priority and a more relaxed sampling time. At each activation tBaseRate checks and unlocks by means of semaphores the $t$ SubRate, which must be executed in the same sampling time. However, since tBaseRate has the highest priority, it continues to execute its jobs, preventing other process executions and completing the elaboration of the fastest part of the model. When it finishes, the previously unlocked $t$ SubRate procedures start to execute their jobs using the CPU on the basis of their priorities.

It should be noted that one of the key concepts which grants the multitasking execution with hard real-time requirements in these control software architectures is the relation between the sampling times of each Task: the base sampling time is decided on the basis of the requirements of the most critical Task, the other Tasks being executed with multiple sampling times with respect to the base sampling time.

Host-Target communications. The Host machine is usually supervised by a general purpose operating system with graphical interfaces, allowing a simple and direct interaction with the user in the design and development phases and for the management of Target-plant interaction. No real-time requirements are necessary: the user prepares its tasks off-line, sets the Target execution, and, at the end, analyzes the obtained data.

Recent technologies allow data exchange between Host and Target using TCP/IP on Ethernet or RS232 protocols. The Target machine is bounded by hard and soft real-time requirements and cannot interrupt its Tasks in a given instant. The Host requires asynchronous mode interaction and the Target reacts as soon as possible, respecting the highest priorities of real-time Tasks. These facts motivate the two fundamental techniques for data exchange:

- "on-the-fly" transfer, in which the Target tries to communicate with the Host during the real-time Task execution; 
- transfer at the end of the current test session, in which, before the tests starts, the Host asks the Target to collect data, the Target executes the test memorizing relevant data, and when each real-time Task ends its jobs and frees the CPU, it transfers the data to the Host.

The first technique does not guarantee that the Host receives all the data related to the real-time signals: the Target could be in a busy state executing a real-time procedure, and it could not manage the communication exchange. This fact can originate an incorrect reconstruction of the observed signals due to data incompleteness.

When Round-robin with Interrupt is used, the transfer job can be executed as a background Task when the ISR does not run; however the ISR can interrupt the communication in any instant, provoking partially data losses. In the RTOS architecture, data collection and transfer to the Host is usually executed by a lowpriority process, which is interrupted by the scheduler when a real-time process has to be executed; obviously, data losses can occur in this case too. Actually this low-priority process can be a web server, and it can manage queries coming from clients all over the net.

The data transfer at the end of the test, according to the second technique, makes possible a correct reconstruction of all signals. This job is not particularly expensive in terms of CPU time for the real-time systems; the Task which manages data storage uses the CPU for brief time intervals, and if the workload of the real-time system is not critical, there is a high probability to complete the job in time. Unlike the first technique, it is possible to correctly reconstruct the acquired signals, paying the price of a retard in the data exchange with the Host, and of growing memory needs.

\section{The prototyping environment proposed in MISTRAL}

In this Section, the software and hardware architecture of a fast prototyping environment developed at the Robotic Laboratory of Politecnico di Torino will be described. It relies on a round-robin with Interrupt architecture and is implemented on a DSP based controller, managed through a Matlab toolbox running on the Host PC.

In Section 4 some experiments and results obtained with this environment will be illustrated.

\subsection{The robotic system}

The experiments were performed on a double-arm planar manipulator with revolute vertical axis joints, sketched in Figure 3.

Two brushless NSK Megatorque direct-drive (i.e. without gearboxes) motors move the joints. The maximum extension of the links $\left(L_{1}+L_{2}\right)$ is about $0.7 \mathrm{~m}$, the angular limits are \pm 2.15 rad for both joints, and the tip height moves parallel to the horizontal plane at a distance of $0.45 \mathrm{~m}$; joint angular positions are measured by internal resolvers.

The two motors are actuated by power drives, which take care of the various and complex functions of these motors and look after the signals coming from the 
Fig. 3. Diagram of the double-link planar manipulator used for testing.

resolvers. The drive communication system deals, in particular, with some of the main features that are basic for control, such as digital input/output signals interchange, application of analog command inputs, and decoding of position information from sensors.

The drive cabinets contain power electronics for the PWM of the motors, and a card devoted to transform analog signals from resolver into digital signals of shaft encoder type, based on a 16 bit microprocessor.

The analog signals coming from the controller are interpreted as torque or velocity reference commands to be applied to the motors, according to the two available control modes: Torque Mode and Velocity Mode. On the basis of the resolver signals, a current loop is closed to control the torque in the first case, whereas an additional velocity loop is added in the second mode. The default mode used to test different types of control algorithms is the Torque mode.

The inner current loop parameters are fixed, and the actuator model can be approximated by a simple proportional gain $K_{v \tau}$ between the input command voltage, $V_{m}$, and the torque $\tau_{m}$ supplied by the motor:

$$
\tau_{m}=K_{v \tau} V_{m}
$$

The optional Velocity Mode is useful in emergency situations, when the user needs to instantly arrest the manipulator motion, pushing the STOP button: a digital input linked to the button activates the velocity control loop, imposing zero velocity reference. The stopping phase will be executed as specified by the internal velocity control algorithm.

The overall plant and the controller can be modelled as in the diagram of Figure 4 , that shows how the controller receives encoders signals and gives back voltage signals in $\mathrm{mV}$, proportional to required command torques. 
Fig. 4. IMI-ODSP model

\subsection{The control system architecture}

The original control system has been replaced by a new one, in which the components for real-time interaction are grouped in a modular industrial standard rack.

This control system environment, called OpenDSP, has been developed by the Mechatronics Laboratory of the Politecnico di Torino and consists of a DSP board and a programmable input/output board. A PLD (Programmable Logic Device) on the latter board allows to configure via software the digital and analog inputs and outputs, and to preprocess these signals in a customized way, before they reach the converters or the DSP. Field interfacing is obtained by means of user customizable boards, packaged with the I/O board and the DSP board in the same rack. The realtime control requirements are guaranteed by the presence of a link between the $\mathrm{I} / \mathrm{O}$ and the DSP boards based on a proprietary bus (called the OpenDSP bus).

The system is linked via enhanced parallel port (EPP) protocol to a desktop PC, working as a Host, and by connections to each axis interface.

A Matlab environment with Simulink runs on the Host PC. The OpenDSP system includes a new toolbox for Matlab called MatDSP, which allows Matlabcode interaction with the DSP. MatDSP too has been developed by the Mechatronics Laboratory of the Politecnico di Torino.

MatDSP makes possible, among other functions, to read and/or change any variable processed by the DSP. For example, the parameters of a control algorithm can be changed "on fly" in a single sampling time in order to guarantee a coherent switch to the new configuration (synchronous mode); or different variables, at user's choice, can be monitored without requiring a more stringent "sample by sample" acquisition (asynchronous mode). It is possible to monitor the real-time variables and the drives status flags, to scope and acquire signals and make any type of mathematical operation on them. A control algorithm written in $\mathrm{C}$ can be compiled, downloaded and started/paused on the DSP. 
Simple graphic user interfaces have been built in the Matlab environment using the GUIDE tool, to simplify testing and management of signals exchanged with the drives. The MatDSP commands have been hidden by a logic construction, grouping the signals in high level functions rather than using them to perform single hardware operations.

For example, a large number of cross-controls is needed to guarantee the correct and safe sequence of operations to enable and start the control task; this would oblige the user to read and change several variables using the primitive statements provided by the MatDSP toolbox. On the contrary, hiding the MatDSP commands under these GUIs allows the user to concentrate on new experiments. An example of one of these GUIs is shown in Figure 5.

Fig. 5. A GUI example for the double arm manipulator supervision.

Three tools are available to the user: the first one, called IMIConsole, is a GUI panel to perform the homing procedure, to prepare and to enable a control algorithm chosen from a list; it is the entry point for the normal interaction with the control system.

The second tool, called IMIExecute, is a GUI panel that allows to select and execute, in single or cyclic mode, a previously planned trajectory and make a home return to the zero position. This GUI shares the same data base of IMIConsole tool to ensure appropriate and safe operations.

The third tool, called IMIReference, does not interact with the system as it is not related to the MatDSP toolbox, unlike the IMIConsole and the IMIExecute GUIs. It is used to generate some simple, basic reference functions, such as joint or cartesian point-to-point moves or circular trajectories, and save them in a MAT file.

From the IMIConsole panel it is possible to open the IMIExecute or the IMIReference GUIs and load a Simulink model of the robot to simulate the planned trajectories before executing them on the real plant. The user can test and change the structure or the paramenters of a control algorithm until a satisfactory response is reached. 
The designer can now translate the algorithm in $\mathrm{C}$ code, compile and download it using the GUIs, impose the same trajectory used in simulation, and enable the robot to execute it. If the experiment is satisfactory the prototyping session ends, otherwise the procedure is repeated with a refined Simulink model or with a new control algorithm.

\subsection{The OpenDSP software architecture}

The OpenDSP real-time software relies on a round-robin with interrupts architecture. When the system is initialized, a main function, Main.c, calls some sub-functions which configure the system on the basis of a group of parameters, some of which fixed and other ones assigned by the user. Then, in an infinite loop two other sub-functions are called in turn: the first one, called Monitor, takes care of data exchange between the Matlab environment and the DSP; the second one, called UserBackground, allows to execute a user code at a lower priority level, which interprets and executes the Matlab commands and interacts with the drives' logic. Both sub-functions have no hard-real-time requirements and can be interrupted when the periodical axis control function, written by the user, starts.

The whole user code is divided in sections and hosted in a file on the basis of a $\mathrm{C}$ written template; no automatic code generation has yet been implemented in this prototyping system. The initial section, the UserInit, contains the code to initialize the customizable characteristics of the system and the starting settings of axis control functions; it is executed one time, when the code downloaded to the DSP is launched. The variables, which must be available in the Matlab workspace, are declared and initialized within this function.

User writes in the subsequentUserISR_INT2 section, the control algorithm code and all the functions useful to close the loop: sensors reading, position reference management and command application. UserISR_INT2 is executed every control sampling time according to the following procedure:

- a timer sends a signal for Start Of Conversion (SOC) to the input and output converters (ADCs and DACs);

- when the conversion ends, a signal for End Of Conversion (EOC) returns, and the DSP stops the current job, i.e., one of the Monitor or UserBackground functions; note that a sampling time delay is inserted by the system in the model of the plant, since the DAC uses the command computed in the previous step;

- UserISR_INT2 is executed, and afterwards the DSP returns to the suspended job.

The sequence assumes that the control algorithm computation ends before the next EOC signal, to allow the execution of portions of non real-time jobs, too. The template is ended by the UserBackground function, that contains the code executed by the DSP when the Monitor and UserISR INT2 functions are inactive. As previously said, this code interprets the commands coming from Matlab and pass them to the DSP environment by means of the Monitor function.

To summarize, the open architecture of this system has allowed to configure five sections of the whole structure: 
- the hardware interface toward the plant, using custom electronics built on a standard development field module to be mechanically compliant with the rack and the stackthrough structure;

- the logical interface between DSP and field modules, managed by the PLD firmware. Starting from a general architecture, the PLD user part is initialized with suitable logic circuits devoted to group and convert signals from and to the field module in registers, or to close faster loops (in microseconds);

- the data base structure of the real-time signals, built in the form of registers and channel manageable by suitable macros in a pre-structured $\mathrm{C}$ header file;

- the Background routine that manages the communication between Host and DSP, and the ISR routine to control the axes, starting from a general and strongly organized C template;

- the asynchronous communication between Matlab user and plant by means of a graphic user interface giving a logical and easier interpretation of the plant functionalities.

\section{Description of a test-Case: prototyping a model-based compensation of nonlinear joint friction}

The model of the manipulator under study [7], [8] can be described by the following second-order nonlinear differential equation:

$$
\mathbf{M}(\mathbf{q}) \ddot{\mathbf{q}}+\mathbf{C}(\mathbf{q}, \dot{\mathbf{q}}) \dot{\mathbf{q}}+\boldsymbol{\tau}_{f}(q, \dot{q})=\boldsymbol{\tau}_{m}
$$

where $\mathbf{q}$, $\dot{\mathbf{q}}$, and $\ddot{\mathbf{q}}$ are the vectors of joint angles, angular velocities and angular accelerations, $\mathbf{M}(\mathbf{q})$ is the configuration-dependent inertia matrix, including both links and motors inertia, $\mathbf{C} \dot{\mathbf{q}}$ is the term containing Coriolis and centrifugal torques, $\boldsymbol{\tau}_{f}$ is the friction torque vector, and $\boldsymbol{\tau}_{m}$ is the command torque vector. No gravity term is present, since the manipulator moves in a horizontal plane. The electrical time-constants of the motors are not considered, as the inner current loop guarantees that they are much faster than the mechanical ones, and that, consequently, the relationship between the input voltage and the output torque is simply given by a known gain $K_{v \tau}$.

The determination of a proper model to describe the friction phenomena, whose effects are modelled in $\boldsymbol{\tau}_{f}$, and the identification of its parameters values have been performed by a series of appropriate tests, and executed by means of an appropriate C-based DSP code, developed within fixed templates. In particular, two different procedures have been applied to perform two different kinds of tests:

- open loop tests (to estimate stiction and friction at high velocity), with the joints free to rotate;

- closed loop tests (to estimate static friction at low velocity, and dynamic friction in the presliding phase), with the manipulator in the controlled configuration.

In particular, starting from the acquired joint position samples and the corresponding velocity values, computed using a simple digital filter, the friction torques have been indirectly derived by considering: 
- in the open loop tests:

$$
\boldsymbol{\tau}_{m, k}=\boldsymbol{\tau}_{f, k}
$$

where $\boldsymbol{\tau}_{m, k}$ and $\boldsymbol{\tau}_{f, k}$ are the $k$-th samples of the applied motor torques and of the joint friction torques, respectively;

- in the closed loop tests at low velocity, as:

$$
\boldsymbol{\tau}_{f}(\dot{\mathbf{q}})+\boldsymbol{\tau}_{e r r}=\boldsymbol{\tau}_{m}-\mathbf{M}(\mathbf{q}) \ddot{\mathbf{q}}-\mathbf{C}(\mathbf{q}, \dot{\mathbf{q}})
$$

from the manipulator dynamic equation (2), where $\tau_{\text {err }}$ is a torque vector that represents all modelling errors and measurement disturbances; such a term has been disregarded, repeating several times the same motion and filtering the measured data to extract the mean values.

Stiction (i.e. friction at zero velocity) has been estimated by tests in which each joint is set in a definite angular position, the drive is set in Torque Mode, and minimal torque increments are supplied in both clockwise $(\mathrm{CW})$ and counterclockwise $(\mathrm{CCW})$ directions. No joint motion is noticeable until the command torque reaches the maximum static friction value. When the joint starts to rotate, the current torque value is registered, and the procedure is repeated for various starting angular positions, to test the stiction dependency on the angular position of the joint.

Tests are executed by means of a DSP code based on a fixed template, modified just in the section relative to the control function, the UserISR_INT2. The command torque increments are supplied in open loop, directly from the user.

The test is executed in the Matlab environment using the IMIConsole GUI to compile and download the real-time code and to enable the axis drives; runtime changes of the command torque reference are allowed by the commands MatDSPvariable (VarName, NewValue) and MatDSPupdate. In particular, the last command lets all the real-time variables, modified by the user with the command MatDSPvariable, be refreshed in the same sampling time.

Finally, the mean stiction value is computed and used as the estimated stiction value.

The contribution of viscous friction at high velocity has been evaluated letting the joints rotate freely, and using the Torque Mode functionality to achieve a situation of dynamic equilibrium at constant velocity, in which the inertial torque is zero, and the friction torque can be assumed to be approximately equal to the command torque.

The DSP code necessary for these experiments is the same used to evaluate stiction, with the addition of the position measurement by means of the macro IOGP_FU1_READ_ENC_CURRENT (Channel) and the acquisition data command, Acquire ( ), at the end of function UserISR_INT2.

This functionality offered by the system is configurable at run-time by the Matlab command MatDSPAcquireConfig (params), choosing: i) which data are to be acquired, ii) data decimation parameters, and iii) the acquisition time interval. It is not an invasive operation for the control function, i.e., it does not cause the violation of the sampling time, because it is executed entirely in the DSP environment to 
avoid a slow data exchange with the PC. The Monitor function returns acquired data to Matlab environment, without real-time constraints, when the user invokes the command MatDSPACqui reLoad ( ) . In the considered case, angular joint position values are acquired for each torque increment. A waiting time interval allows the end of the acceleration fluctuations, after which a two seconds acquisition is started. Angular velocity data are computed from the measured positions, for each joint and for each rotation direction, and for every velocity sample the corresponding friction torque is assumed equal to the command torque $\boldsymbol{\tau}_{m}$. The velocity data obtained have a lower bound value of about $2 \mathrm{rad} / \mathrm{s}$, due to the sudden transition from stop to motion and viceversa.

Joint friction at low velocity has been then investigated by an experimental session performed with the manipulator in the controlled configuration. A simple PD control law is used to assign to each joint the position/velocity profile defined by the user, to properly collect data for the estimation of static friction at low velocity, and dynamic friction in the presliding phase. More code is added at the UserISR_INT2 to supply a micro-interpolation mechanism for the user profile, together with a section devoted to the position data processing needed by the PD algorithm. The IMIExecute GUI is used, together with the IMIConsole, to transfer the reference position vector to the DSP running code, which interpolates and executes the movement. The user provides the reference vector and the data acquisition request by means of the IMIExecute, and then, after a pre-positioning phase, the task is executed and a MAT file containing the acquired data is saved in a predefined directory.

On the basis of the acquired data, the well-known LuGre model [11], [13] has been considered to represent the friction torques on each joint of the manipulator. Such a model includes both a steady-state (static) friction curve, and the dynamic friction behavior during the presliding phase by means of a "bristle" model, according to the following equations:

$$
\begin{aligned}
& \frac{\mathrm{d} z_{i}}{\mathrm{~d} t}=\dot{q}_{i}-\frac{\left|\dot{q}_{i}\right|}{g_{i}\left(\dot{q}_{i}\right)} \sigma_{0, i} z_{i} \\
& \tau_{f, i}=\sigma_{0, i} z_{i}+\sigma_{1, i} \frac{\mathrm{d} z_{i}}{\mathrm{~d} t}+f_{i}\left(\dot{q}_{i}\right)
\end{aligned}
$$

where $z_{i}$ is a state variable representing the average bristle deflection for joint $i$, $\sigma_{0, i}$ and $\sigma_{1, i}$ are model parameters that are assumed to be constant, and functions $g_{i}\left(\dot{q}_{i}\right)$ and $f_{i}\left(\dot{q}_{i}\right)$ model the Stribeck effect and the viscous friction, respectively. For constant velocity, the steady-state friction torque is then given by:

$$
\tau_{f, i_{s s}}=g_{i}\left(\dot{q}_{i}\right) \operatorname{sgn}\left(\dot{q}_{i}\right)+f_{i}\left(\dot{q}_{i}\right)
$$

Among the different parameterizations that can be used to describe $g_{i}\left(\dot{q}_{i}\right)$ and $f_{i}\left(\dot{q}_{i}\right)$, the following ones have been chosen because they fit well the acquired data:

$$
\begin{aligned}
g_{i}\left(\dot{q}_{i}\right)= & \alpha_{0, i}+\alpha_{1, i} \mathrm{e}^{-\frac{\dot{q}_{i}}{\dot{q}_{s 1, i}} \operatorname{sgn}\left(\dot{q}_{i}\right)} \\
& +\alpha_{2, i}\left(1-\mathrm{e}^{-\frac{\dot{q}_{i}}{\dot{q}_{s 2, i}} \operatorname{sgn}\left(\dot{q}_{i}\right)}\right) \\
f\left(\dot{q}_{i}\right)= & \alpha_{3, i} \dot{q}_{i}+\alpha_{4, i} \dot{q}_{i}^{2}
\end{aligned}
$$


The static parameters in (8) and (9) (i.e., the four $\alpha_{k, i}$ 's for each joint, together with $\dot{q}_{s 1, i}$ and $\dot{q}_{s 2, i}$ ), have been estimated by considering tentative values between 0.1 and $0.3 \mathrm{rad} / \mathrm{s}$ for the exponential parameters $\dot{q}_{s 1, i}$ and $\dot{q}_{s 2, i}$ (on the basis of the acquired data), and applying a least square algorithm to a linearized expression of (7)-(9) to estimate the $\alpha$ 's parameters for each joint. By some iterations, the values reported in Table 1 have been obtained.

Table 1. Estimated static parameters of the LuGre friction model.

\begin{tabular}{|c|c|c|c|c|}
\hline & $\begin{array}{c}\text { Joint 1 } \\
\omega>0\end{array}$ & $\begin{array}{c}\text { Joint 1 } \\
\omega<0\end{array}$ & $\begin{array}{c}\text { Joint 2 } \\
\omega>0\end{array}$ & $\begin{array}{c}\text { Joint 2 } \\
\omega<0\end{array}$ \\
\hline$\alpha_{0}$ & 40.854 & -46.473 & 17.837 & 3.408 \\
\hline$\alpha_{1}$ & -32.454 & 53.873 & -14.837 & -0.408 \\
\hline$\alpha_{2}$ & -31.233 & 55.738 & -14.998 & -0.635 \\
\hline$\alpha_{3}$ & -0.760 & -0.293 & -0.156 & -0.104 \\
\hline$\alpha_{4}$ & -0.262 & 0.177 & -0.050 & 0.036 \\
\hline$\dot{q}_{s 1}$ & 0.19 & 0.14 & 0.2 & 0.3 \\
\hline$\dot{q}_{s 2}$ & 0.17 & 0.15 & 0.19 & 0.1 \\
\hline
\end{tabular}

Figure 6 shows the resulting steady-state friction torque together with the acquired data for the first joint (for positive and negative velocities). Similar results have been obtained for the second joint.

Fig. 6. Friction torque $(\mathrm{Nm})$ on joint 1 for positive and negative velocities $(\mathrm{rad} / \mathrm{s})$.

Following a procedure similar to the one proposed in [12], under some pre-sliding assumptions, the dynamic friction parameters $\sigma_{0, i}$ and $\sigma_{1, i}$ have been estimated, computing $z_{i}$ by integrating equation (5) from joint position measures, acquired during an appropriate motion of each joint. In particular, a slowly growing torque ramp has been applied to each joint to estimate $\sigma_{0, i}$, and a torque step to estimate $\sigma_{1, i}$ (see [12] for details). A good approximation of such parameters could be obtained only starting from high precision joint position measurements. Since in our case the encoder signal is decoded with a resolution of only $2 \pi / 76800 \mathrm{rad}$, a subsequent model validation phase has been performed, by comparing the real robot behavior 
with the results of some simulation tests, carried out by a Simulink model, which can be directly run from the IMIConsole GUI. Some adjustments of the estimated values of the dynamic friction parameters have been allowed by this procedure, but some additional investigation will be necessary. The currently estimated values are reported in Table 2.

Table 2. Estimated dynamic parameters of the LuGre friction model.

\begin{tabular}{|l|c|c|c|c|}
\hline & $\begin{array}{l}\text { Joint 1 } \\
\omega>0\end{array}$ & $\begin{array}{c}\text { Joint 1 } \\
\omega<0\end{array}$ & $\begin{array}{l}\text { Joint 2 } \\
\omega>0\end{array}$ & $\begin{array}{c}\text { Joint 2 } \\
\omega<0\end{array}$ \\
\hline$\sigma_{0}$ & 55500 & 26000 & 12600 & 12600 \\
\hline$\sigma_{1}$ & 1000 & 800 & 70 & 70 \\
\hline
\end{tabular}

Preliminary tests have been performed to evaluate the improvements that can be obtained from the control point of view by friction compensation. The applied inverse dynamic control scheme, including only static friction compensation (as the currently available dynamic friction estimation is not yet satisfactory), is of the following type:

$$
\boldsymbol{\tau}_{m}=\mathbf{M}(\mathbf{q})\left(\ddot{\mathbf{q}}_{r}-\mathbf{v}_{c}\right)+\mathbf{C}(\mathbf{q}, \dot{\mathbf{q}}) \dot{\mathbf{q}}+\hat{\boldsymbol{\tau}}_{f}(\dot{\mathbf{q}})
$$

where $\ddot{\mathbf{q}}_{r}$ is the joint acceleration reference vector, $\hat{\boldsymbol{\tau}}_{f}(\dot{\mathbf{q}})$ is the estimated friction torque vector, and a PD control algorithm has been considered to define the outer loop law $\mathbf{v}_{c}$. The corresponding DSP code is very similar to the one used in the low velocity friction estimation tests; sub-sections, containing the robot inverse dynamics and an high-order polynomial function, approximating the estimated static friction model, have been simply added within the UserISR_INT2.

Figure 7 shows the time-history of the resulting position error of the first joint, for a circular, cartesian reference trajectory, defined by means of the IMIReference GUI, when $\hat{\boldsymbol{\tau}}_{f}(\dot{\mathbf{q}})=\mathbf{0}$ is considered, i.e. without any friction compensation, and when $\hat{\boldsymbol{\tau}}_{f}(\dot{\mathbf{q}})$ corresponds to the estimated steady-state friction curve. As Figure 7 shows, even if only static friction has been compensated, a significant error reduction has been obtained (similar results have been achieved for the second joint, too).

\section{Conclusion and Research Perspectives}

Rapid prototyping systems are used to speed up the development of a final product. In industrial robotics these systems have real-time requirements fulfilled by means of architectures which allow the designer to concentrate his work on the prototype development.

At the Politecnico di Torino a solution based on the well known round-robin with Interrupt software architecture, the OpenDSP system, has been proposed within the MISTRAL project. The hardware architecture is based on a Host-Target solution, 
Fig. 7. Inverse dynamic control without and with friction compensation: position error on joint 1 .

with the Host running Matlab and a dedicated toolbox to manage the Target DSP board.

Some experiments devoted to model and control the friction phenomena have been presented, demonstrating a right tradeoff between usability and efficiency of the OpenDSP system.

In perspective, an advanced prototyping architecture based on standard real-time operating systems will be investigated and implemented, aimed at providing an extended environment, with increased interaction capabilities between data acquisition, model analysis and control design.

Particular attention will be devoted to integrate discrete state/event transitions and continuous control, extended use of graphical modelling (under Simulink), automatic code generation, vision and force sensors integration. From this point of view an interesting real-time operating system is Linux with RTAI patch. RTAI (Real-Time Application Interfaces) [15] provides Linux with real-time features (appropriate syscalls, RT scheduling, reduced latency, etc.) and allows, in its present development state, the automatic code generation from Matlab Real-Time Workshop.

\section{Acknowledgements}

The work described in this Chapter has been funded by MIUR under Mistral Project. Parts of the work have been co-funded by the Italian Space Agency (ASI). A particular thank goes to CSPP-LIM, Politecnico di Torino, for technical support.

\section{References}

1. C.J. Boots, and G.P. Kurpis, The New IEEE Standard Dictionary of Electrical and Electronic Terms [Inluding Abstracts of All Current IEEE Standards], fifth edition, New York: IEEE, 1993. 
2. Jane W.S. Liu, Real-Time systems, Prentice-Hall, Upper Saddle River, New Jersey, 2000.

3. David E. Simon, An Embedded Software Primer, Addison-Wesley, 2001.

4. Ken Arnold, Embedded Controller Hardware Design, LLH Technology Publishing, 2001.

5. Andrew S. Tanenbaum, Modern Operating Systems, 2nd Edition, Prentice Hall, 2001.

6. F. Kordon, and Luqi, "An Introduction to Rapid System Prototyping", IEEE Transactions on Software Engineering, vol. 28, NO. 9, September 2002.

7. B. Bona, M. Indri, and N. Smaldone, "Open System Real-Time architecture and software design for robot control", IEEE/ASME International Conference on Advanced Intelligent Mechatronics (AIM01), Como, Italy, pp. 349-354, 2001.

8. B. Bona, M. Indri, and N. Smaldone, "An Experimental Setup for Modelling, Simulation and Fast Prototyping of Mechanical Arms", IEEE Conference on Computer-Aided Control Systems Design, Glasgow, UK, pp. 207-212, 2002.

9. H. Hanselmann, "Automotive control: from concepts to experiments to product", IEEE Int. Symp. on Computer-Aided Control Systems Design, Dearborn, Michigan, pp. 129134, 1996.

10. G. Mason, A. Pongpunwattana, and M. Berg, "Design and modeling of a flexible test bed for use in control system analysis and verification”, Mechatronics, vol. 12, pp. 891-904, 2002.

11. C. Canudas de Wit, and H. Olsson and K.J. Åström and P. Lischinsky, "A New Model for Control of Systems with Friction”, IEEE Trans. on Automatic Control, vol. 40, n. 3, pp. 419-425, 1995.

12. C. Canudas de Wit, and P. Lischinsky, "Adaptive Friction Compensation with partially Known Dynamic Friction Model," Int. J. of Adaptive Control and Signal Processing, vol. 11, pp. 65-80, 1997.

13. H. Olsson, K.J. Åström, C. Canudas de Wit, M. Gäfvert, and P. Lischinsky, "Friction Models and Friction Compensation", European Journal of Control, n. 4, pp. 176-195, 1998.

14. L. Sciavicco, and B. Siciliano, Modelling and Control of Robot Manipulators, 2nd Edition, Springer-Verlag, London, UK, 2000.

15. http://www.aero.polimi.it/ ${ }^{\text {rtai/. }}$ 